Acta Psychologica 27 (1967) 71-74; (C) North-Holland Publishing Co., Amsterdam Not to be reproduced by photoprint or microfilm without written permission from the putlisher

\title{
THE EFFECT OF SIGNAL INTENSITY AND TRAINING ON SIMPLE REACTION TIME
}

\author{
3. KORNBLUM \\ Mental Health Research Institute, University of Michigan, Ann Arbor, USA \\ and \\ W. G. KOSTER \\ Institute for Perception Research, Eindhoven, The Netherlands
}

\begin{abstract}
A discrepancy between data obtained by the two authors under apparently identical conditions lead to the execution of a simple experiment during the symposium. The results make it probable that a difference in stimulus intensity was the cause of the discrepancy. A plea is made for the advantages of small symposia for the rapid and exciting exchange of information.

In a presentation to the symposium on Attention and Performance held at Driebergen in August 1966, Koster reported data for two subjects (KOSTER and BEKKER, 1966) confirming DAvts' recent findings (1965) on the psychological refractory period in that no significant delays were found for the response to the second of two closely spaced signals when the first signal consisted of a subject-initiated response. In Koster's experiment the subject initiated a trial by pressing a key which started the temporal sequence of a trial. After a variable interval this trigger response was followed by a visual signal to which the subject was instructed to respond as quickly as possible by pressing anothar key. The visual signal was always the same requiring no choice, and the time intervals ranged between 10 and 1000 msec. The results which are plotted in fig. 1 clearly indicate that the delay in the response to the visual signal as a function of the interval all but disappeared after very little training indeed.

During the discussion which followed Koster's presentation, Kornblum noted that in a study which he had recently completed with 34 subjects (KoRNBLUM and EMERSON, 1966) and in which Davis' self-initiating procedure had been used, the data he obtained had faiied to confirm Davis' findings. Kornblum's results differed from Davis' and Koster's in two important respects: (1) a significant delay of the response to the visual signal was obtainc 1 ar: the short intervals, and (2) training reduced the RT at the long intervals leaving the RT at the short intrrvals unaffected (see fig. 2a). This, in the light of Koster's evidence which had provided independent verification for the 'absence of a delay', set the stage for the puzzle. How
\end{abstract}




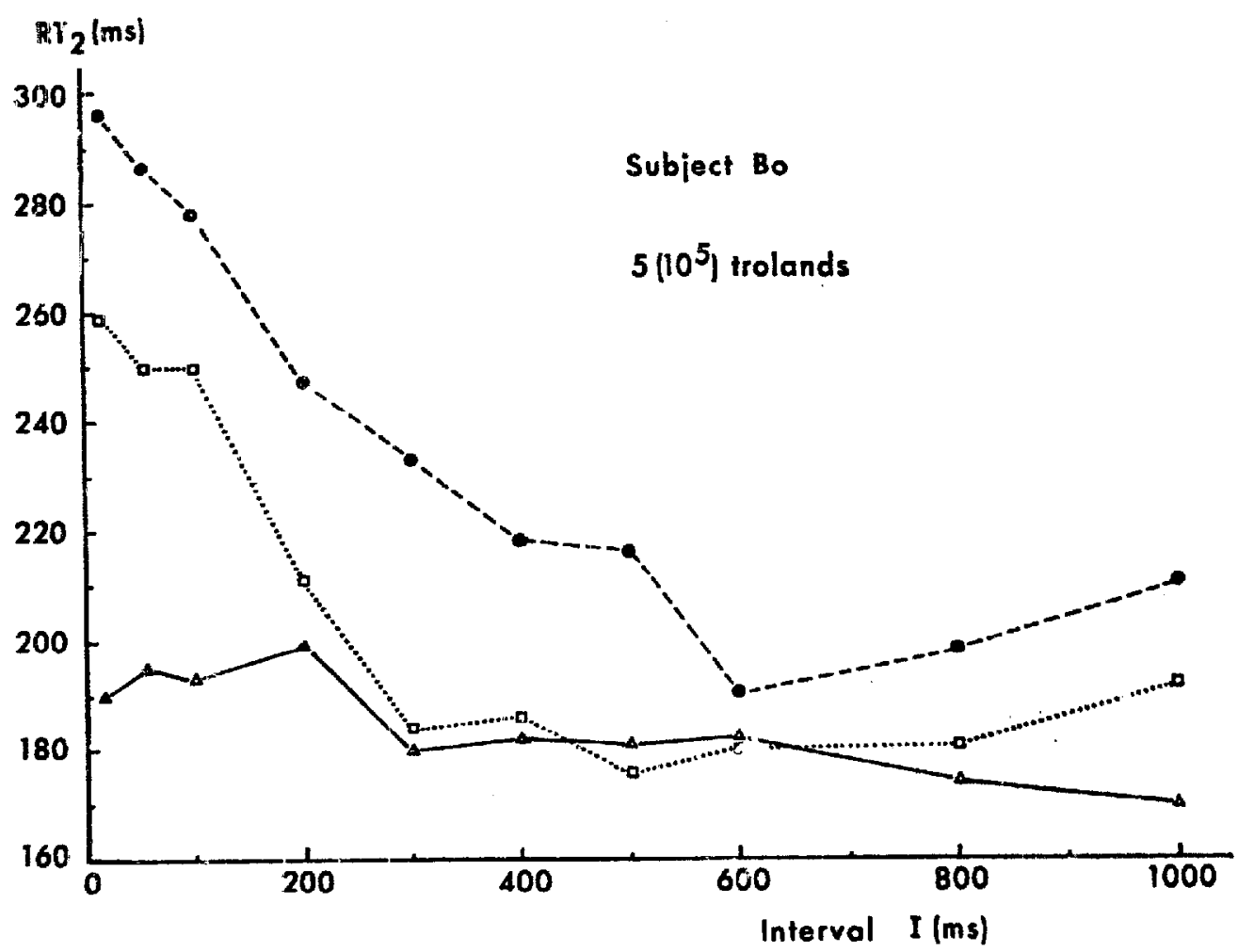

Fig. 1. The RT to the second signal is plotted on the ordinate as a function of the interval between the self-initiated response and the visual signal. Each point represents the median value of about 4 observations for one subject. The series represent successive stages in training ( $\ldots_{-\ldots}$ first series, $\square \ldots \square$ second series, $\triangle-\triangle$ third series).

could two apparently identical experimental procedures produce two such reliable yet contradictory sets of data? Obviously, what had been taken to be 'identical experimental procedures' were not in fact so.

Arl opportunity to ascertain the precise manner in which our experiments differed was conveniently provided on the following day when, as part of the sympusium programme, a visit was scheduled to the Institute of Perception Research in Eindhoven, where Koster's laboratory was located. His proceaures could, therefore, be examined in detail and at leisure. Asice from the fact that Koster's subjects were not naive to the task and that his interval programmes were not quite homogeneous, the single most strikingly different feature of his experiment was the luminance of the visual signal---Koster's signals were presented at a retinal illumination of $5\left(10^{5}\right)$ trolends for a 20 wsec duration. Furthermore, while it is perfectly true that Davis' signals were neon lamps and could, therefore, come nowhere near this intensity level, Davis' subjects viewed the signals through a $3 \mathrm{ft}$ cardboard tube which would have made for a high contrast between the signal/no-signal conditions. Kornblum, on the other hand, who had also used neon bulbs, had the subjects view the signals against a grey background at a distance of $6 \mathrm{ft}$ and 
the whole experinient took place in reduced ambient room illumination. Thus, on empirical grounds it appeared that signal luminance might well account for the differences between our results. Koster was able to verify this hypothesis on the very next day by running one subject on his own equipment, using his own procedure, and reducing the retinal illumination to 50 trolands. The data (fig. 2 b) for that subject showed the same trend
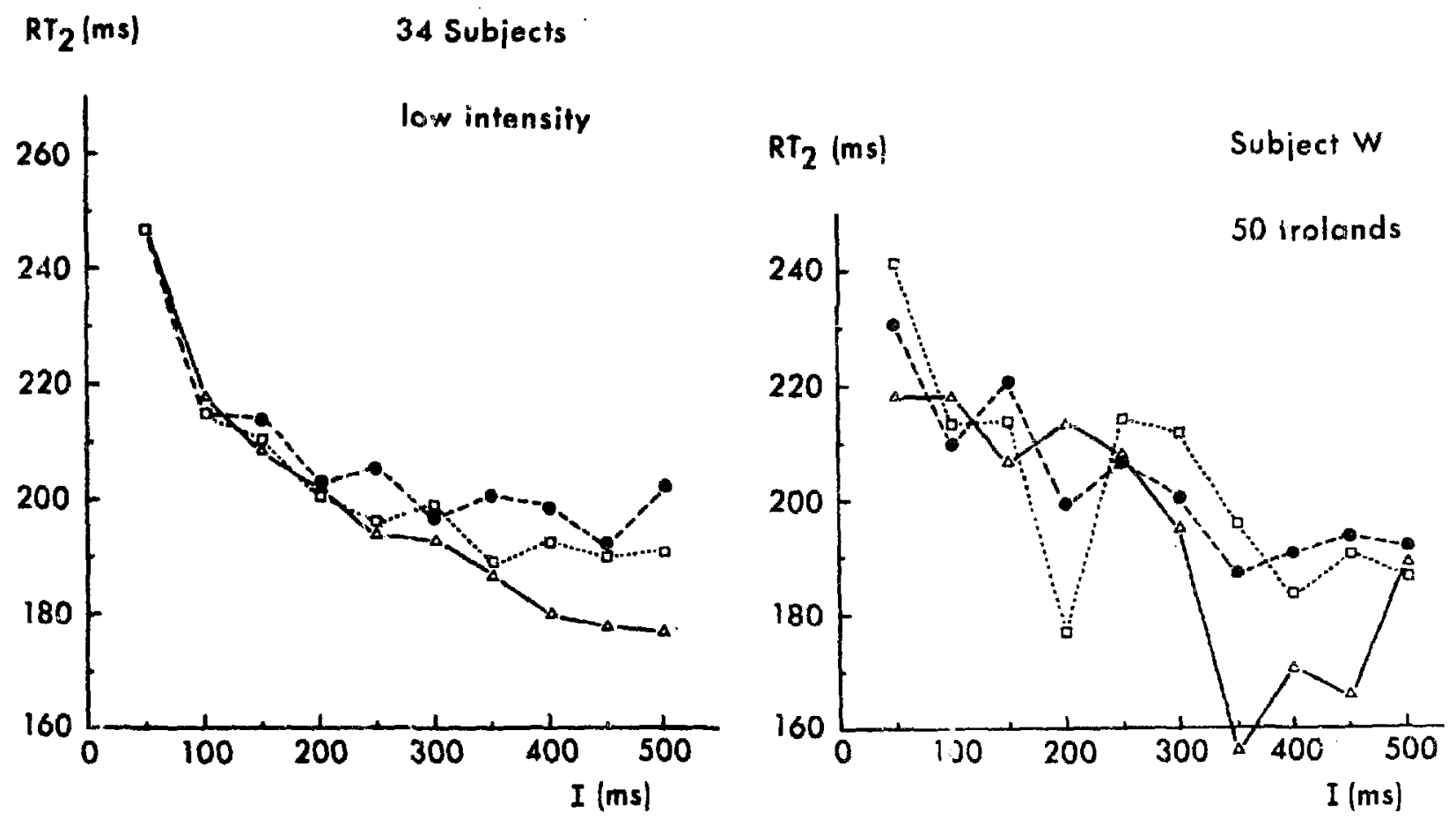

Fig. 2. The RT to the sucond signal is plotted on the ordinate as a function of the interval between the self-initiated responise and the visual signal. The series represent successive st iges in training (-... first series, $\square \ldots \square$ second series, $\triangle-\triangle$ third series). In fig. 2a each point represents 10 observations for each of 34 subjects, for a total of 340 observations per point. In fig. $2 b$ each point represents 3 observations for one subject.

as Kornblum's findings. The dicrepancy between our results had, therefore, been reconciled; or, more accurately, the reason for the differences between our results had tentatively been traced to an important difference in our procedure. ${ }^{1}$

Clearly additional work must be done to verify this findings and, once verified, the question of how signal luminance can be made to account for this effect must be seriously posed. Many conjectures in this respect are possible, however, it is not the object of this note to entertain any of them

1 While this article was in press Kornblum $f . i l e d$ to replicate the original Davis and Koster results in his own laboratory using a bright signal as is suggested in this note. 
at this stage; to do so would in a sense violate the intent of this communication which is meant to be quite informal. We have attempted to relate a sequence of events as they occurred during the course of a three day symposium which was organized around a narrowly circumscribed set of topics for a small number of participants. These, in addition to other important factors, contributed to a sustained high level of enthusiasm ihroughout the three days of which this small incident is a direct, and for lis particularly satisfying outcome. Whether our results will be verified in future work, of course, remains to be seen. What is certain, however, is the vast superiority of such small symposia or 'w/orking retreats' over mammoth conventions, for the rapid and exciting exchange of information.

\section{REFERENCES}

Davis, R, 1965. Quart. J. exp. Psychol., 17, 75-78.

KornBLUM, S. and P. Emerson, 1966. Manuscript in preparation. Ku ster, W. G. and J. A. M. BekKer, 1966. This volume, p. 64. 